\title{
Co-pollinators and specialization in the pollinating seed-consumer mutualism between senita cacti and senita moths
}

Received: 19 February 2002 / Accepted: 7 August 2002 / Published online: 19 September 2002

(C) Springer-Verlag 2002

\begin{abstract}
Specialization of a plant on a particular pollinator may not evolve if co-pollinators are effective and abundant. This is particularly evident if fruit set is resource limited and cannot be increased above the levels produced by the actions of co-pollinators. The pollinating seed-consuming interaction between senita cacti and senita moths in the Sonoran Desert presents a paradox because it exhibits many traits resembling those of the highly specialized yucca/yucca moth system, but also involves co-pollinators. For 6 years, we studied how contributions of nocturnal senita moths and diurnal co-pollinating bees to fruit set depended on resource and pollen limitation, time of flower closing, and the onset and phenology of flowering. Fruit set was typically resource limited. Fruit set of flowers exposed only to senita moths was not different from resource-limited fruit set of control flowers. When only co-pollinating bees were allowed to visit flowers, however, fruit set became pollen limited. Only in one year when fruit set was pollen limited were bees able to increase fruit set beyond the level resulting from senita moth pollination. High temperatures commonly induced flowers to close before sunrise so that diurnal bees were unable to visit flowers. This was particularly important from 1998 to 2000, when flowering did not begin until late in spring when temperatures were already high enough to induce flowers to close before sunrise. Bees were typically functionally redundant with senita moths; excluding bees from visiting flowers did not alter fruit set. Nevertheless, extreme specialization of floral traits to exclude co-pollinators has not evolved in senita, possibly because there are times when bees do increase fruit set. This can occur when senita moths are rare, fruit set is pollen limited, cool tem-
\end{abstract}

J.N. Holland $(\bowtie) \cdot$ T.H. Fleming

Department of Biology, University of Miami, P.O. Box 249118,

Coral Gables, Florida 33124, USA

Present address:

J.N. Holland, Department of Ecology and Evolutionary Biology, University of Arizona, Tucson, Arizona 85721, USA,

e-mail: jholland@email.arizona.edu,

Tel.: +1-520-6213534, Fax: +1-520-6219190 peratures prevent flowers from closing before sunrise, and flowering begins early in spring.

Keywords Co-pollinator · Mutualism · Pollination · Senita $\cdot$ Functional redundancy

\section{Introduction}

Generalized pollination systems are more common than specialized ones. This is due in part to spatio-temporal variation in abundant, effective pollinators and spatiotemporal variation in plant abundance and food rewards (Waser et al. 1996). Yet, highly specialized plant-pollinator systems do exist. Two well-known cases are yucca/ yucca moth (Pellmyr et al. 1996) and fig/fig wasp (Bronstein 1992; Herre 1996) interactions, where pollinating insects lay their eggs in flowers. In fig and yucca systems, co-pollinators are virtually absent, plants and insects obligately depend on one another, species are apparently coevolved, and most insects pollinate actively, using behavioral and morphological traits for pollen collection and its placement on stigmas (Bronstein 1992; Pellmyr et al. 1996; Pellmyr 1997).

Several other pollinating seed-consuming interactions occur, but none is as specialized as fig and yucca mutualisms. These systems include globeflowers and flies (Pellmyr 1989, 1992; Hemborg and Despres 1999; Jaeger et al. 2000), Lithophragma and Greya moths (Thompson and Pellmyr 1992; Thompson 1994; Pellmyr and Thompson 1996), Silene vulgaris and Hadena moths (Pettersson 1991a, 1991b, 1992a, 1992b), and senita cacti and senita moths (Fleming and Holland 1998; Holland and Fleming 1999a, 1999b). Evolution of extreme specialization of these plants on pollinating seed-consumers may depend on presence or absence of co-pollinators (Pettersson 1991a; Thompson and Pellmyr 1992; Pellmyr and Thompson 1996). For example, the positive effects of Greya moth pollination on Lithophragma reproduction can be minimized when co-pollinators are abundant (Thompson and Pellmyr 1992). Also, evolution of plant 
specialization on a pollinator may not occur if there is little reproductive benefit to eliminating co-pollinators (Aigner 2001).

The mutualism between senita cacti and senita moths presents an apparent paradox. It exhibits traits of the specialized fig and yucca systems, including behavioral and morphological traits of an active pollinator. Yet, senita are similar to plants of less specialized systems because flower traits do not completely exclude co-pollinators. A few bee species pollinate flowers of senita cacti (Fleming and Holland 1998). How specialized are senita and senita moths on one another? Senita moths are, to the best of our knowledge, specialized and obligately dependent on senita. They do not visit flowers of other cacti and all of their life stages are associated with senita (Holland and Fleming 1999a). There still remains some question, however, as to how specialized and dependent senita are on senita moths, given the presence of copollinators. Here, we attempt to determine how dependent senita reproduction is on senita moth pollination and when co-pollinators may contribute. We studied how the contribution of senita moths and bees to senita reproduction depends on resource-limited fruit set, and the daily and seasonal phenology of flowers.

\section{Materials and methods}

Study species and study site

Senita cacti (Lophocereus schottii) and senita moths (Upiga virescens; Pyralidae) are endemic to the Sonoran Desert of North America. Two species occur within Lophocereus, but only senita has been studied in detail. Senita moths are the only nocturnal pollinator of senita cacti, but some bees [Apis mellifera (Apidae); Augochlorella, Agapostemon, Dialictus spp. (Halictidae)] visit flowers after sunrise. Senita flowers are whitish-pink, self-incompatible, and hermaphroditic (one stigma and 100 anthers). Anthers dehisce when flowers open at sunset. Flowers close within $16 \mathrm{~h}$. Flowering can start in late March and may last to September. Fruit set (fraction of flowers initiating fruit development) is limited by rainfall and water availability (Holland 2002).

In 1995, 1996, and 1998-2000, we studied senita cacti near Bahia de Kino (BK), Sonora, Mexico $\left(28^{\circ} \mathrm{N}, 111^{\circ} \mathrm{W}\right)$. At BK we had two study sites, Seri Flats and Polilla Flats. In 1997, we worked at Organ Pipe Cactus National Monument (ORPI) in Arizona $\left(31^{\circ} \mathrm{N}, 112^{\circ} \mathrm{W}\right)$. For 20 years preceding 1994 , mean annual rainfall at $\mathrm{BK}$ was $126.4 \pm 72 \mathrm{~mm}( \pm 1 \mathrm{SD})$ (DICTUS, Bahia de Kino, Sonora, Mexico). Aside from two major rain events (one before the 1995 flowering season and one before the 2000 flowering season), only trace amounts of rain occurred from 1995 to 2000 . We do not have a quantitative record of rainfall after 1995 because BK's weather station was removed in 1994. Nevertheless, our qualitative record and the production of very few flowers and leaves in 1999 and 2000 by creosote (Larrea tridentata), a plant robust to drought stress (Reynolds et al. 1999), indicate years of progressive drought.

Resource- versus pollen-limited fruit set

The contribution of senita moths and bees to fruit set may depend on whether fruit set is limited by pollen or resources. We supplemented flowers with pollen in 1995, 1997, 1998, 1999, and 2000 to measure resource-limited fruit set and whether fruit set was limited by pollen. We measured fruit set for two groups of plants, one with open-pollinated flowers and the other with pollen-supplemented flowers (10-30 flowers per plant, 5-11 plants per group). Stigmas of pollen-supplemented flowers were provided an excess of pollen from $~ 50$ anthers of a fresh flower from another plant Pollen was gently brushed onto stigmas within a few hours after flowers opened. Pollen was easily applied to stigmas and adhered without disrupting flowers. Fruit set for open- and hand-pollinated flowers were compared using $t$-tests.

We conducted an additional pollen supplementation experiment in May and July 1998 following the approach of Zimmerman and Pyke (1988). This allowed us to assess pollen limitation and whether plants differentially allocate resources to flowers that have received excess pollen. We measured fruit set for 10-30 open-pollinated and 10-30 pollen-supplemented flowers on experimental plants ( $n=8$ plants) and 10-30 open-pollinated flowers on control plants ( $n=8$ plants). We used Wilcoxon matched-pairs signed-ranks tests and Wilcoxon rank sum tests to compare fruit set among these three sets of flowers (Sokal and Rohlf 1995).

\section{Fruit set from senita moths and bees}

We conducted pollinator exclusion experiments to assess the potential of senita moths and bees to contribute to fruit set. Flowers within plants were haphazardly assigned as control, diurnal, or nocturnal. Control flowers were exposed to nocturnal and diurnal pollinators. Senita moths were excluded from diurnal flowers by covering flowers with bridal-veil netting at night. Bees were excluded from nocturnal flowers by covering flowers with netting before sunrise. We quantified fruit set for 100-600 flowers per treatment across 7-11 plants. Treatments were applied from late April to mid-May in 1995 and 1996 at BK, in 1997 at ORPI, and in May and June in 1998 at BK. We compared fruit set among treatments for each trial using repeated measures ANOVAs (SAS Institute 1999). We report Greenhouse-Geisser adjusted $P$-values. We re-analyzed 1995, 1996, and 1997 data (Fleming and Holland 1998; Fleming et al. 2001) to account for among-plant variance and to compare results with resource limitation. Trials were not conducted in 1999 and 2000 because flowers closed prior to sunrise, naturally excluding diurnal bees.

\section{Daily and seasonal phenology of flowers}

If the onset of flowering is delayed until late spring (e.g., due to water stress), if temperatures are sufficiently warm in late spring, and if warm temperatures induce flowers to close before sunrise, then bees could be naturally excluded for an entire flowering season. We monitored the onset and phenology of flowering by counting flowers on a weekly basis in 1995, 1996, and 1998-2000 at Seri Flats ( $n=20$ plants) and in 1998-2000 at Polilla Flats ( $n=30$ plants). In 1998 we studied whether temperature affected time of flower closing by placing a digital thermometer by a focal flower of 27 plants. We recorded the temperature at which a flower closed and maximum and minimum temperatures during the time a flower was open. We scored flowers as open until wilting petals would not allow pollinator entry. Flower closing versus temperature was analyzed using linear regression. We monitored temperature changes during 1998, 1999, and 2000 seasons at Seri Flats by recording maximum, minimum, and average daily temperatures using a Hobo temperature data logger. The data logger was elevated to $2 \mathrm{~m}$ in an open-bottom box that blocked direct sunlight.

\section{Results}

\section{Resource- versus pollen-limited fruit set}

Fruit set did not differ between pollen-supplemented flowers and open-pollinated flowers in four of six com- 
Table 1 Fruit set (percent of flowers initiating fruit development) of senita cacti from 1995 to 2000 at Bahia de Kino $(B K)$ and 1997 at Organ Pipe Cactus National Monument (ORPI) for pollen-supplemented flowers, open-pollinated flowers, flowers where diurnal co-pollinating bees were excluded (i.e., flowers only exposed to senita moths), and flowers where nocturnal senita moths were excluded (i.e., flowers only exposed to diurnal bees). Values are mean fruit set $( \pm 1 \mathrm{SE} ; n=7-15$ plants); means within a year followed by different letters are significantly different (Tukey's test, $P<0.05)$

\begin{tabular}{llllllll}
\hline Treatment & BK 1995 & BK 1996 & ORPI 1997 & BK May 1998 & BK June 1998 & BK 1999a & BK 2000a \\
\hline Pollen-supplemented flowers & $41 \pm 16$ & - & $39 \pm 4$ & $39 \pm 9$ & - & $47 \pm 11$ & $66 \pm 4$ \\
Open-pollinated flowers & $49 \pm 7$ A & $46 \pm 6$ A & $25 \pm 2$ A & $49 \pm 7$ A & $33 \pm 4$ A & $51 \pm 11$ & $55 \pm 8$ \\
Flowers exposed only to moths & $37 \pm 7$ AB & $42 \pm 6$ A & $17 \pm 3$ B & $52 \pm 7$ A & $25 \pm 8$ A & - & - \\
Flowers exposed only to bees & $20 \pm 5$ B & $8 \pm 3$ B & $6 \pm 2$ C & $4 \pm 2$ B & $0.5 \pm 0.5$ B & - & - \\
\hline
\end{tabular}

a Pollinator-exclusion experiments were not conducted because flowers closed before sunrise. Presumably, fruit set from control openpollinated flowers in 1999 and 2000 resulted from nocturnal pollination by senita moths

Table 2 Mean $( \pm$ SE) fruit set (percent of flowers initiating fruit development) in May and July 1998 for senita cacti for hand-pollination (outcrossed) and open-pollination flowers on experimental plants ( $n=8$ plants) and open-pollination flowers on control unmanipulated plants $(n=8$ plants)

\begin{tabular}{llr}
\hline Treatment & May & \multicolumn{1}{l}{ July } \\
\hline Pollen-supplemented experiment plants & $38.9 \pm 9.0$ & $22.5 \pm 6.6$ \\
Open-pollinated experiment plants & $41.9 \pm 5.9$ & $0.0 \pm 0.0$ \\
Open-pollinated control plants & $56.5 \pm 8.1$ & $0.0 \pm 0.0$ \\
\hline
\end{tabular}

parisons (Table 1): $1995(t=0.507, d f=15, P>0.05)$, May 1998 ( $t=0.964, d f=17, P>0.05), 1999(t=0.329, d f=12$, $P>0.05)$, and $2000(t=1.351, d f=26, P>0.05)$. Pollinators were abundant enough to account for as high fruit set as senita had resources to mature. At ORPI in 1997, fruit set of open-pollinated flowers was lower than fruit set of pollen-supplemented flowers (Table $1 ; t=3.053, d f=16$, $P<0.05)$, indicating that pollen was limiting fruit set. Fruit set was also pollen limited in July 1998, when no open-pollinated flower initiated fruit (Table 2). In May 1998, fruit set did not differ between any pairwise comparison among hand-pollinated flowers on experimental plants, open-pollinated flowers on experimental plants, and open-pollinated flowers on control plants (Table 2; $P>0.05$ for all comparisons). This is consistent with fruit set being limited by resources, and indicates that senita cacti did not allocate more resources to flowers receiving excess pollen.

\section{Fruit set from senita moths and bees}

Pollinator exclusion experiments had a significant effect on fruit set (Table 1) in 1995 at $\mathrm{BK}\left(F_{2,18}=6.05\right.$, $P<0.01), 1996$ at BK $\left(F_{2.18}=21.40, P<0.0001\right), 1997$ at ORPI $\left(F_{2.16}=41.44, \quad P<0.0001\right)$, May 1998 at $\mathrm{BK}$ $\left(F_{2,20}=43.44, \quad P<0.0001\right)$, and June 1998 at BK $\left(F_{2,20}=23.92, P<0.0003\right)$. Fruit set of control open-pollinated flowers remained fairly constant from 1995 to 2000 at BK, ranging from $46 \%$ to $55 \%$ (Table 1), with a $7 \%$ coefficient of variation $(\mathrm{CV})$. At $\mathrm{BK}$, fruit set of flowers exposed only to senita moths was also relatively constant $(\mathrm{CV}=16 \%)$, but fruit set of flowers exposed only to bees was highly variable $(\mathrm{CV}=129 \%)$.

Effects of exclusion treatments on fruit set were similar among trials in 1995, 1996, May 1998, and June 1998, but effects of exclusion treatments from the 1997 trial differed from other trials. In 1997, excluding bees from flowers, such that flowers were only pollinated by senita moths, significantly reduced fruit set from openpollinated control flowers, because fruit set was pollen limited (Table 1). Excluding moths from visiting flowers, such that only bees pollinated flowers, also significantly reduced fruit set from open-pollinated flowers, because fruit set was pollen limited. Fruit set of flowers only exposed to bees was significantly less than fruit set of flowers only exposed to senita moths. Results from 1997 indicate that, due to pollen limitation, pollination by both moths and bees was necessary to account for control open-pollinated fruit set.

In 1995, excluding bees from flowers did not significantly reduce fruit set from open-pollinated flowers (Table 1). When moths were excluded from visiting flowers, however, fruit set significantly declined and shifted from being resource to pollen limited. Fruit set when moths were excluded was not different from fruit set when bees were excluded, suggesting possible multiplicative or additive effects of moths and bees on open-pollinated fruit set. As in 1995, exclusion of bees did not reduce fruit set from open-pollinated flowers in 1996 (Table 1). Also as in 1995, exclusion of senita moths in 1996 reduced fruit set from open-pollinated flowers, suggesting that fruit set again became pollen limited. In contrast to 1995 , however, in 1996 fruit set of flowers exposed only to moths was greater than fruit set of flowers exposed only to bees. From 1995 to 1996, there was a reduction in fruit set of flowers visited only by bees $\left(F_{1,9}=8.73\right.$, $P<0.01)$, indicating that bee visits had declined. In May and June 1998, fruit set of flowers exposed only to senita moths did not differ from fruit set of open-pollinated flowers. Fruit set of flowers exposed only to diurnal bees was significantly less than fruit set of open-pollinated flowers and flowers exposed only to senita moths. Again, when only bees were allowed to visit flowers, fruit set shifted from resource to pollen limited. Bee contributions to fruit set declined from May to June in 1998. 
Fig. 1 Phenology of flower production (no. flowers per plant, mean $\pm 1 \mathrm{SE}$ ) for senita cacti at our Seri Flats study site from 1995 to 1996 (Holland and Fleming 1999a) and 1998-2000 (A-E) and at our Polilla Flats study site from 1998 to $2000(\mathbf{F}-\mathbf{H})$ near Bahia de Kino, Sonora, Mexico ( $n=20-30$ plants). Apr April, Jun June, Jul July, Aug August
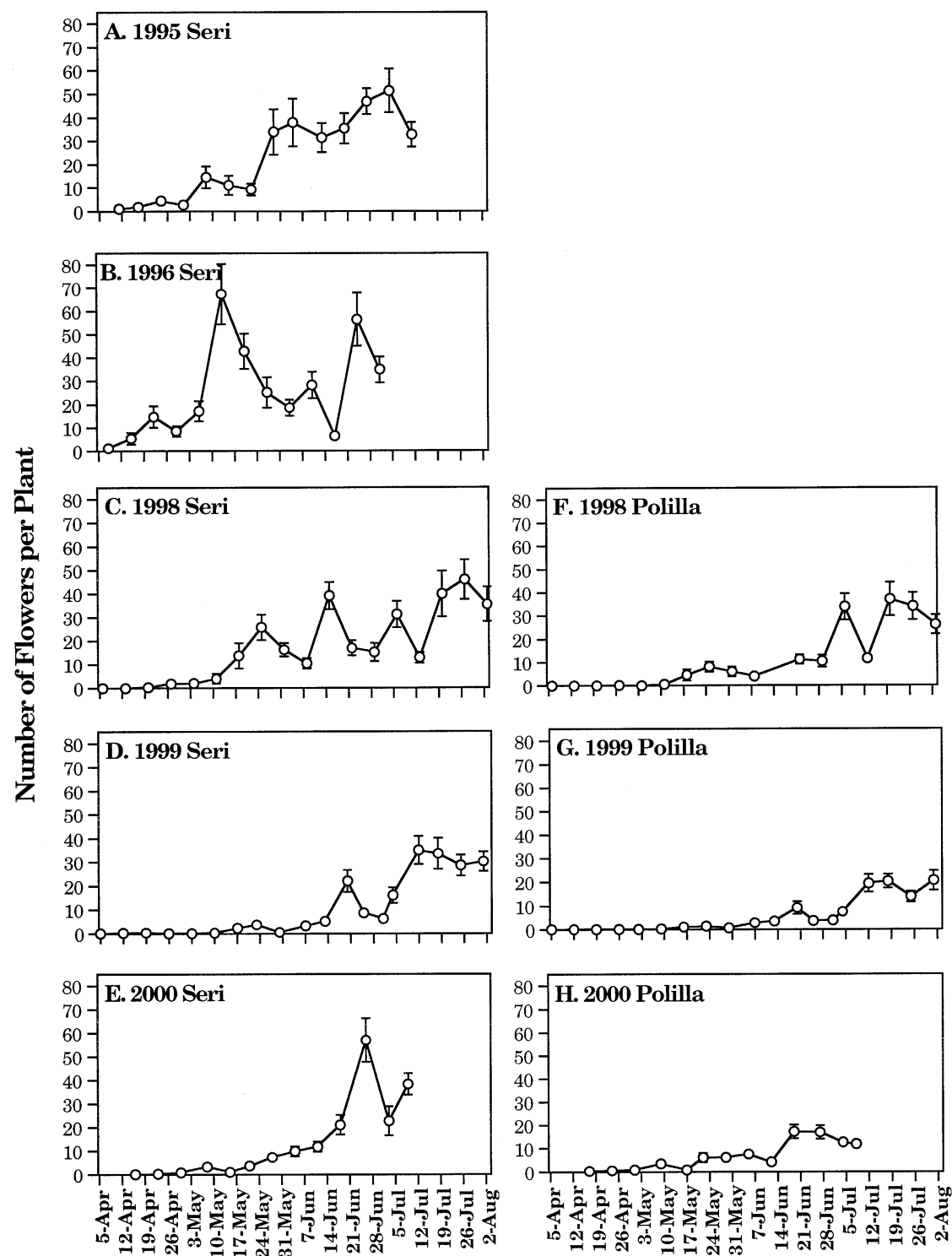

In 1999 and 2000, pollinator exclusion experiments were not conducted because flowers closed before sunrise, precluding bee visitation. We assume that $100 \%$ of the resource-limited fruit set in 1999 and 2000 resulted from pollination by senita moths.

Daily and seasonal phenology of flowers

In order for bees to contribute to fruit set, the onset of a flowering season must occur when bees are available to visit flowers and flowers must remain open past sunrise. From 1995 to 2000 at both BK sites, the onset of flowering occurred progressively later in spring (Fig. 1). In 1995 and 1996, flowering began by mid-April. In 1998, however, flowering did not begin until late May and in 1999 and 2000 not until mid-June. Flowering began slightly earlier in 2000 than 1999, likely due to the first heavy rain since 1994 on 6 March 2000 before flowering of 2000. Whether flowers closed before or after sunrise was determined by ambient temperature (Fig. 2; $\left.F_{1,25}=120.13, P<0.0001\right)$. As the minimum nightly temperature increased from sunset to sunrise, flowers closed sooner after they opened. Temperature explained $83 \%$ of variation in time of flower closing. Because the minimum, maximum, and average daily temperatures increase as flowering seasons progress from April through August (Fig. 3), bees were naturally excluded from visiting flowers after mid-May to early June. Bees were naturally excluded from visiting flowers for entire seasons of 1999 and 2000, because flowering did not begin until after temperatures were already high enough that flowers closed before sunrise. 


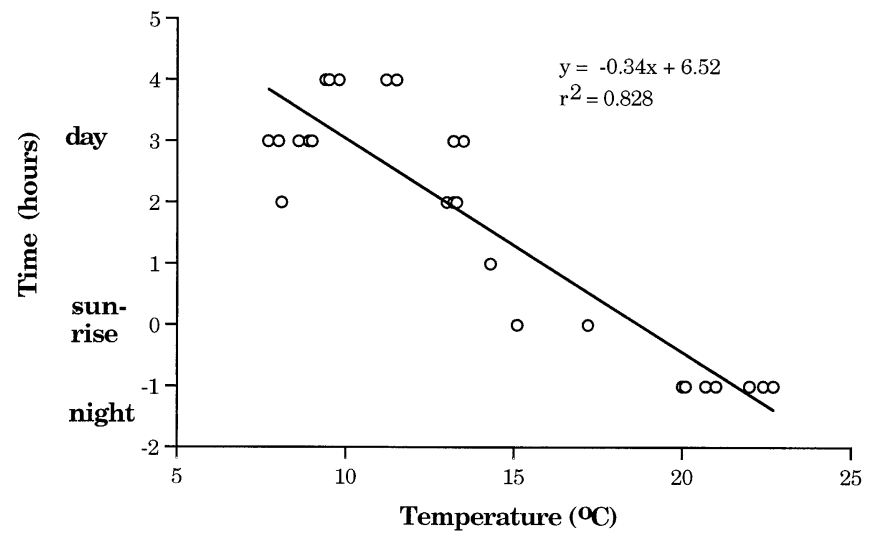

Fig. 2 Time that senita cactus flowers closed ( $n=27$ plants) as a function of ambient temperature. Time is expressed on an hourly basis relative to sunrise, where time at sunrise is zero, times after sunrise are positive values, and times before sunrise are negative values. Values for temperature are the minimum temperatures measured during the night
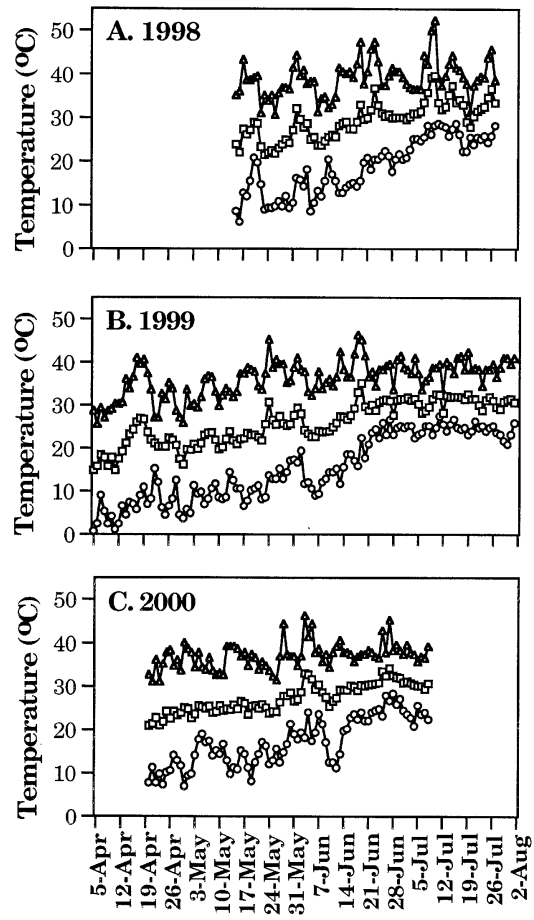

Fig. 3 Maximum $(\Delta)$, mean $(\square)$, and minimum $(\bigcirc)$ daily temperatures during flowering seasons of 1998 (A), 1999 (B), and 2000 (C) at Seri Flats in Bahia de Kino, Sonora, Mexico. For abbreviations, see Fig. 1

\section{Discussion}

For 6 years, we studied how the effect of co-pollinating bees on reproduction of senita cacti, which has a highly specialized obligate pollinator (senita moths), depended on resource- and pollen-limited fruit set, time of flower closing, and onset of flowering seasons. It is difficult to assess the importance of bees because senita are longlived (50-80 years) with $>20$ reproductive years, and within a single year, can flower for 3-5 months. Also, biotic and abiotic features of the Sonoran Desert can vary greatly in the short and long term. We interpret the importance of bees for senita reproduction under the assumption that these six years of data capture both shortand long-term influences on senita moths, bees, and senita cacti.

Halictid and honey bees were the only co-pollinators. During the first hours after sunrise, they collected pollen from senita flowers, but were never observed harvesting nectar. Bee visits to senita are likely spill over from visits to flowers of saguaro (Carnegiea gigantea) and organ pipe (Stenocereus thurberi) cacti. Bees contribute more to fruit set of these cacti than to that of senita (Fleming et al. 2001), likely because bees are attracted to the large quantities of pollen and nectar produced by flowers of the bat-pollination syndrome of saguaro and organ pipe. Unlike visits to saguaro and organ pipe, whose flowers remain open long after sunrise, bees were not always able to visit senita flowers. Warm nocturnal temperatures commonly caused flowers to close before sunrise, likely due to wilting caused by evaporation of water from petals. Because flowers typically close before sunrise after late May, bees are unable to visit senita flowers when the onset of flowering is delayed due to drought stress. Bees can visit senita flowers from April to mid-May when typically cool temperatures do not cause nocturnal flower closing. When only bees pollinated flowers, fruit set became pollen limited and dropped by $60-99 \%$, depending on the year. It was only under pollen-limited conditions of 1997 that bees increased fruit set beyond that resulting from senita moth pollination. Hence, bees can affect fruit set only under certain environmental and ecological conditions. These conditions include years or populations when senita are not water stressed so that flowering begins early in spring; when temperatures are cool enough for flowers to remain open after sunrise; and, when senita moths are rare, so that fruit set is pollen-limited.

When senita moths are abundant enough that fruit set is limited by resources (water, Holland 2002), co-pollinating bees are functionally redundant. A component of a system is functionally redundant if it can be removed from that system without altering a function or process (e.g., fruit set). First, fruit set was usually unaltered when bees were excluded from flowers, indicating that senita moths could account for $100 \%$ of fruit set. Second, senita moths were usually abundant enough that adding pollen above their pollination did not increase fruit set permitted by limited resources. Only in two periods of our studies was fruit set pollen limited: in 1997 at ORPI, when moths were not abundant, and in July 1998, when no flower set fruit because senita moths were in pre-adult life stages between moth cohorts. Note that lack of fruit set in the absence of senita moths confirms that other nocturnal insects do not pollinate flowers (Holland and Fleming 
1999a). Third, senita moths always visit flowers before bees. Senita cacti do not increase resource allocation to initiate fruit of flowers with excess pollen, as may occur when both moths and bees visit same flowers. Also, no differences occur in seeds per fruit between senita mothand bee-pollinated flowers (Fleming et al. 2001). Hence, bees are commonly, but not always, functionally redundant with senita moths. Fruit set limited by resources prevents bees from increasing fruit set above pollination by senita moths. The exact opposite occurs between Greya moths and co-pollinators of Lithophragma. Resource limitation can prevent Greya moths from increasing fruit set beyond that resulting from co-pollinators (Thompson and Pellmyr 1992).

The question remains as to how specialized senita cacti are on senita moths and whether natural selection is likely to lead to the evolution of traits that exclude copollinators. Floral and reproductive traits of Lophocereus schottii appear highly divergent from those of their closest relatives. $L$. schottii and $L$. gatesii, along with three species of Pachycereus, occur in a monophyletic clade (Gibson and Horak 1978; Cornejo and Simpson 1997). In fact, $L$. schottii and $L$. gatesii are now being placed in the genus Pachycereus and renamed as $P$. schottii and $P$. gatesii (Hunt and Taylor 1990; Anderson 2001; A. C. Gibson, personal communication). Pachycereus is an ancestrally bat-pollinated genus. Most species of Pachycereus produce large flowers (7-12 cm long) and nectar volumes (up to $2 \mathrm{ml}$ ) that attract vertebrate pollinators. Pachycereus marginatus is hummingbird pollinated and senita's closest relative (Gibson and Horak 1978; Cornejo and Simpson 1997; Hartman et al., in press). It has diurnal, red flowers 4-5 cm long. In contrast, senita has nocturnal, whitish-pink flowers $2-4 \mathrm{~cm}$ long; most flowers produce no nectar, and those that do contain $\sim 0.5 \mu \mathrm{l}$ (Holland and Fleming 1999a). Senita does not attract other moth pollinators probably because most flowers produce no nectar. The shift from bat pollination in $P$. marginatus and Lophocereus was likely a single evolutionary event (Hartmann et al., in press), but the evolutionary shift to nocturnal insect pollination in Lophocereus is likely novel, reflecting some degree of specialization on senita moths.

Despite some apparent specialization on senita moths, senita cacti have not evolved floral traits that exclude all other pollinators. Senita cacti may be in an evolutionary transition between a less specialized system with co-pollinators, like the Lithophragma/Greya system, and a highly specialized system, like the yucca mutualism. On the other hand, co-pollinating bees may limit evolution of extreme specialization of senita cacti on senita moths. Given that bees are functionally redundant with senita moths, but can contribute to fruit set under certain conditions, how might bees influence evolution of floral traits of senita cacti? In order for floral traits to evolve that exclude co-pollinating bees, there must be a cost to reproduction associated with maintaining bee pollinators (Aigner 2001). In other words, a mutant plant which excludes bees must have greater reproduction. Hence, bees may contribute to reproduction, but that contribution must be less than the increase in reproduction associated with excluding bees. It may be that selection favors traits that result in floral specialization on senita moths, but at the same time flowers retain traits that allow co-pollinators to contribute to cactus reproduction in years or populations when fruit set is pollen limited (cf. Mayfield et al. 2001). On the other hand, because senita reproduction is limited by water resources, selection could favor traits in cacti that increase water-use efficiency and alleviate resource-limited fruit set (cf. Galen et al. 1999). For example, selection may favor reduced water allocation to flower petals to maintain turgor pressure after sunrise. This water could be allocated to increase resource-limited fruit set, in the process eliminating access to flowers for co-pollinating bees. Similarly, selection may favor continued reduction in nectar secretion; water in nectar could be allocated to increase fruit set. In either case, selection on floral traits and the inclusion or exclusion of co-pollinators likely varies in space and time depending on both biotic (moth and bee abundance) and abiotic (rainfall, temperature) conditions.

In conclusion, features of both plants and pollinators contribute to how a particular pollinator influences plant reproduction and natural selection (Pellmyr and Thompson 1996; Galen et al. 1999; Aigner 2001). Yet, these features are only rarely static. Instead, they vary in space and time as changes occur in the environmental and ecological context in which interactions take place. For interactions between senita cacti and co-pollinating bees, traits of senita cacti, such as flower phenology, time of flower closing, and resource-limited fruit set, contribute to the minimal effect of bees on cactus reproduction. These features of senita cacti, and their influence on bee contributions to fruit production, varied both within and among years depending to a large degree on the water resources in the environment.

Acknowledgements We thank the Mexican government and the U.S. National Park Service for study permits. Field work was assisted by K. Blanco Cisneros, K. Clark, M. Olivo Hernandez, D. Kiley, M. Lee, C. Marschner, J. Massoud, L. Newton, and H. Passmore. We thank F. Molina-Freaner for continued support and assistance in Mexico. Comments by J. L. Bronstein, N. B. Davies, D. L. DeAngelis, C. C. Horvitz, M. V. Price, S. T. Schultz, and N. M. Waser improved this paper. Research was supported by NSF grants DEB-9421673 and DEB-9800775, National Geographic Society, and a Grant-in-Aid of Research from National Academy of Sciences and Sigma Xi. Preparation of this paper was made possible by the National Parks Ecological Research Fellowship Program, a program funded by the National Park Foundation through a generous grant from the Andrew W. Mellon Foundation.

\section{References}

Aigner PA (2001) Optimality modeling and fitness trade-offs: when should plants become pollinator specialists? Oikos 95: 177-84

Anderson EF (2001) The cactus family. Timber Press, Portland, Ore.

Bronstein JL (1992) Seed predators as mutualists: ecology and evolution of the fig/pollinator interaction. In: Bernays EA (ed) 
Insect-plant interactions, vol IV. CRC Press, Boca Raton, Fla. pp 1-44

Cornejo DO, Simpson BB (1997) Analysis of form and function in North American columnar cacti (Tribe Pachycereeae). Am J Bot 84:1482-1501

Fleming TH, Holland JN (1998) The evolution of obligate pollination mutualisms: senita cactus and senita moth. Oecologia 114:368-375

Fleming TH, Sahley CT, Holland JN, Nason JD, Hamrick JL (2001) Sonoran Desert columnar cacti and the evolution of generalized pollination systems. Ecol Monogr 71:511-530

Galen C, Sherry RA, Carroll AB (1999) Are flowers physiological sinks or faucets? Costs and correlates of water use by flowers of Polemonium viscosum. Oecologia 118:461-470

Gibson AC, Horak KE (1978) Systematic anatomy and phylogeny of Mexican columnar cacti. Ann Miss Bot Garden 65:999-1057

Hartmann S, Nason JD, Bhattacharya D (in press) Phylogenetic origins of Lophocereus (Cactaceae) and the senita cactus - senita moth pollination mutualism. Am J Bot

Hemborg AM, Despres L (1999) Oviposition by mutualistic seedparasitic pollinators and its effects on annual fitness of singleand multi-flowered host plants. Oecologia 120:427-436

Herre EA (1996) An overview of studies on a community of Panamanian figs. J Biogeogr 23:593-607

Holland JN (2002) Benefits and costs of mutualism: demographic consequences in a pollinating seed-consumer mutualism. Proc R Soc Lond B 269:1405-1412

Holland JN, Fleming TH (1999a) Mutualistic interactions between Upiga virescens (Pyralidae), a pollinating seed-consumer, and Lophocereus schottii (Cactaceae). Ecology 80:2074-2084

Holland JN, Fleming TH (1999b) Geographic and population variation in pollinating seed-consuming interactions between senita cacti (Lophocereus schottii) and senita moths (Upiga virescens). Oecologia 121:405-410

Hunt DR, Taylor NP (1990) The genera of Cactaceae: progress towards a consensus. Bradleya 8:85-107

Jaeger N, Till-Bottraud I, Despres L (2000) Evolutionary conflict between Trollius europaeus and its seed-parasite pollinators Chiastocheta flies. Evol Ecol Res 2:885-896

Mayfield MM, Waser NM, Price MV (2001) Exploring the "most effective pollinator principle" with complex flowers: bumblebees and Ipomopsis aggregata. Ann Bot 88:591-596
Pellmyr O (1989) The cost of mutualism: interactions between Trollius europaeus and its pollinating parasites. Oecologia 78:53-59

Pellmyr O (1992) The phylogeny of a mutualism: evolution and coadaptation between Trollius and its seed-parasitic pollinators. Biol J Linn Soc 47:337-365

Pellmyr O (1997) Pollinating seed eaters: why is active pollination so rare? Ecology 78:1655-1660

Pellmyr O, Thompson JN (1996) Sources of variation in pollinator contribution within a guild: effects of plant and pollinator factors. Oecologia 107:595-604

Pellmyr O, Thompson JN, Brown JM, Harrison RG (1996) Evolution of pollination and mutualism in the yucca moth lineage. Am Nat 148:827-847

Pettersson MW (1991a) Flower herbivory and seed predation in Silene vulgaris (Caryophyllaceae): effects of pollination and phenology. Holarct Ecol 14:45-50

Pettersson MW (1991b) Pollination by a guild of fluctuating moth populations: option for unspecialization in Silene vulgaris. J Ecol 79:591-604

Pettersson MW (1992a) Taking a chance on moths: oviposition by Delia flavifrons (Diptera: Anthomyiidae) on the flowers of bladder campion, Silene vulgaris (Caryophyllaceae). Ecol Entomol 17:57-62

Pettersson MW (1992b) Density-dependent egg dispersion in flowers of Silene vulgaris by the seed predator Hadena confusa (Noctuidae). Ecol Entomol 17:244-248

Reynolds JF, Virginia RA, Kemp RR, Soyza AG de, Tremmel DC (1999) Impact of drought on desert shrubs: effects of seasonality and degree of resource island development. Ecol Monogr 69:69-106

SAS (1999) SAS version 8.1. SAS Institute, Cary, N.C

Sokal RR, Rohlf FJ (1995) Biometry. Freeman, New York

Thompson JN (1994) The coevolutionary process. University of Chicago Press, Chicago, Ill.

Thompson JN, Pellmyr O (1992) Mutualism with pollinating seed parasites amid co-pollinators: constraints on specialization. Ecology 73:1780-1791

Waser NM, Chittka L, Price MV, Williams NM, Ollerton J (1996) Generalization in pollination systems, and why it matters. Ecology 77:1043-1060

Zimmerman M, Pyke GH (1988) Reproduction in Polemonium: assessing the factors limiting seed set. Am Nat 131:723-738 